\title{
A Computational Model for Processing Object Motion Based on Retina Neural Mechanism*
}

\author{
Zhi-hua Hu, Xiao Luo, Zhong-ze Chen \\ University of South China, Hengyang, Hunan Province, P. R. China \\ zzchen801@163.com.
}

\begin{abstract}
In this paper, a bioinspired neural model for detecting object motion based on retina computational machanism is proposed based on synthesizing representative works on modeling retina and incorporating some latest findings on retina mechanisms. To understand more in-depth the retina machanism of motion detection, the spatio-temporal properties of each type of neurons in the retina motion pathway are then mathematically analyzed. And then a set of experiments and quantitative analysis of the experimental results are accomplished for assessing the model performance on motion detection. Experimental results show the effectiveness of this proposed model on detecting object motion.

Index Terms - retina neural mechanism, retina neural network, object motion detection, computational model.
\end{abstract}

\section{I . Introduction}

The ability to detect motion in the visual scene is a fundamental computation in the visual system that is firstly performed in the retina[1]. As for complete models of retina motion extracting channel, Boahen etc. proposed[2 3] a retinomorphic system, which uses four different kinds of neurons for firing specific spikes in response to concrete stimuli and representing the input data. F. Barranco[4] designed and implemented an event-driven processing scheme based on artificial retinas for the detection of spatiotemporal features. Stephen A. Baccus[5] etc. investigated the circuit basis for detecting object motion by recording intracellularly from all classes of retinal interneurons while simultaneously recording the spiking output of many ganglion cells.

These findings and works sketch the structural scheme of motion processing channel in retina. However, mathematical analysis of the models and their applications to motion processing are insufficient without exception. Fortunately, numerous neurophysiological findings[1-3][6] on retina information processing pathways have been revealed. These new findings provide great source of ideas for our modeling the retina more reasonably.

So mechanism as well as models of computing object motion in retina incorporating these latest findings will be dealt with.

This paper is organized as following. Model for processing object motion based on retina mechanism is firstly proposed in section 2 . In section 3, experimental results and corresponding analysis is implemented. Finally, conclusions on the proposed algorithm are drawn out in section 4 .

\section{A Model for Processing Object Motion Based on Retina Neural Mechanism}

In this section, a neural organization structure for processing object motion based on retina mechanism will be described at first. Then the spatio-temporal properties of each type of neurons in the motion pathway will be proposed and analyzed in succession.

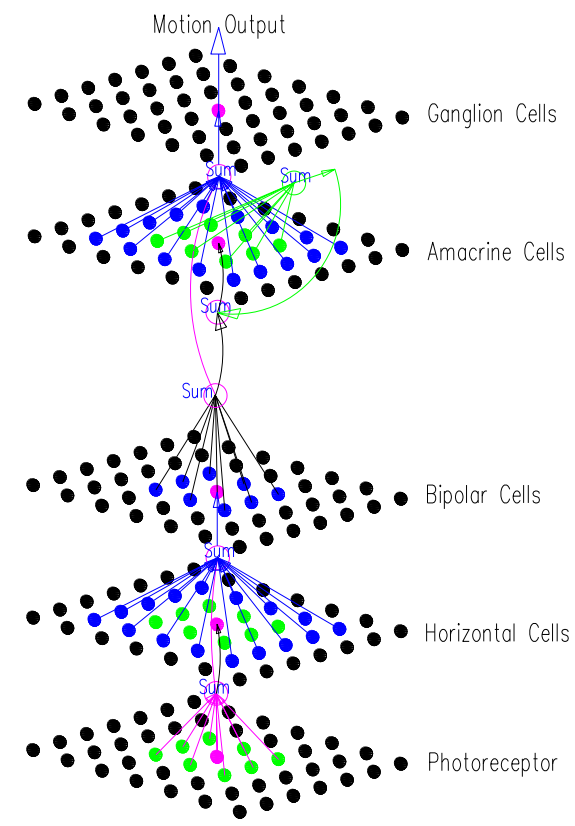

Fig.1 The wiring diagram of the cells which constitute retina motion pathway

\section{A. A Model for Processing Object Motion Based on Retina Neural Mechanism}

Experimental data [7] and theoretical analysis [8-9] show that directional selectivity is the result of local, postsynaptic, nonlinear interactions in the DS(directionally selective) cell. The light responses of cones and bipolar cells are not directionally selective[10]. The direction-selective circuit in the retina relies highly upon the selective wiring of synaptic contacts between SAC(starburst amacrine cell) distal dendritic tips and the dendrites of the DS GCs(ganglion cells). As a presynaptic interneuron, the SAC is asymmetrically connected to DS cells and delivers direct inhibition to DSGCs. The SACs

\footnotetext{
*This work was supported by Grant 09JJ6096, awarded by the Hunan Natural Science Foundation, China, and Grant 2007FJ4154, awarded by the China Hunan Provincial Science \& Technology Department.
} 
pointing in the null direction deliver inhibition and those pointing in the preferred direction do not. They project inhibition laterally ahead of a stimulus moving in the null direction [11]. In addition, starburst inhibition is itself directionally selective: it is stronger for movement in the null direction [11]. Excitation in response to movement in null direction is reduced by an inhibitory signal acting at a site that is presynaptic to the DS cell. Thereby excitation is reduced by enhanced inhibition given stimulus of motion in the null direction, and thus the effect of directional selectivity is realized. Therefore, signaling between the cones, bipolar cell, SAC, and DSGC constitutes a neural network that generates the DS light responses of the DSGC[12]. The retina motion pathway, in a form of laminar organization, is shown as Fig.1.

\section{B. Spatio-Temporal Properties of Cone Cells}

Photoreceptor cells sample optical signals and convert the light information into biological signals. Some studies[11] have shown that the conversion is nonlinear processing and the input-output dynamic characteristics of photoreceptor cells can be described as mathematical formulas [7]:

$$
O_{p}(x, y, t)=\mu_{p} I_{p}(x, y, t) k_{p}(x, y, t)
$$

Where $O_{p}(x, y, t)$ represents the output of the light-sensitive cell $(x, y)$ at time point $t, I_{p}(x, y, t)$ for the brightness distribution, $k_{p}(x, y, t)$ denotes the pigment concentration, and between $I_{p}(x, y, t)$ and $k_{p}(x, y, t)$ there exists a relationship as equation:

$$
\tau_{p} \frac{d}{d t} k_{p}(x, y, t)+\left(1+\mu_{p} I_{p}(x, y, t)\right) k_{p}(x, y, t)=1
$$

Where the parameter $\tau_{p}$ represents the time constant for updating the pigment, and $\mu_{p}$ denotes the pigment bleaching constant.

\section{Spatio-Temporal Properties of Horizontal Cells}

Horizontal cells accept the input of the photoreceptor cells[8], acting as a low-pass filter in the transmission of information from responses of receptors[11].

When suitable parameters are given, the receptive field of a horizontal cell can be modeled [13] as:

$$
W_{h}\left(x, y ; x_{0}, y_{0}\right)=w_{h} \exp \left(-\frac{\left(x_{0}-x\right)^{2}+\left(y_{0}-y\right)^{2}}{2 \sigma_{h}^{2}}\right)
$$

Where $w_{h}$ represent the amplitudes of the Gaussian weight components, whose standard deviations is $\sigma_{h}$, in $W_{h}\left(x, y ; x_{0}, y_{0}\right)$. And $\left(x_{0}, y_{0}\right)$ represent a neighbor area of cell $(x, y)$.

Signals converge into horizontal cell $\left(\mathrm{x}_{s} \mathrm{y}\right)$ is:

$$
I_{h}(x, y)=\sum_{x_{0}, y_{0} \in R_{h}} W_{h}\left(x, y ; x_{0}, y_{0}\right) O_{p}\left(x_{0}, y_{0}\right)
$$

Where $O_{p}\left(x_{0}, y_{0}\right)$ represents the output signals from a photoreceptor located at $\left(x_{0}, y_{0}\right)$ in the $R_{h}$ of cell $(x, y)$.

The response dynamics of a horizontal cell $(x, y)$ to its stimulus is described as following equation [7]:

$$
\tau_{h} \frac{d}{d t} O_{h}(x, y . t)-\gamma^{2} \frac{d^{(2)}}{d t^{2}} O_{h}(x, y . t)+\left[1+\lambda I_{h}(x, y, t)\right] O_{h}(x, y . t)=\lambda I_{h}(x, y, t)
$$

Where $O_{h}(x, y, t)$ is the output of a horizontal cell $(x, y)$, and $I_{h}(x, y, t)$ the input of the cell; parameter $\gamma$ is the extended length of excitement field, $\tau_{h}$ time constant of the horizontal cell, and $\lambda$ the feedback factor.

\section{Spatio-Temporal Properties of Bipolar Cells(BCs)}

Bipolar cells are the only ones with receptive field properties that match the requirements for the essential part of motion computation of nonlinear spatial summation over small subunits in the ganglion cell receptive field center.

Center-surround antagonistic receptive field (CSARF) organization is the basic synaptic circuit for spatial information processing in the visual system. BCs are the first neurons along the visual pathway that exhibit CSARF organization. In both the receptive field center and the antagonistic surround region, the bipolar cell's temporal filter followed a biphasic time course. The surround response was delayed and inverted in sign relative to the center.

Motion in the object region drives these bipolar cells, and their outputs are rectified before summation by the ganglion cell [14-15].

And the weight distribution in bipolar receptive field can be denoted as:

$$
\begin{aligned}
& W_{b}\left(x, y ; x_{0}, y_{0}\right)= \\
& w_{b 1} e^{-\frac{\left(x_{0}-x\right)^{2}+\left(y_{0}-y\right)^{2}}{2 \sigma_{b 1}{ }^{2}}}-w_{b 2} e^{-\frac{\left(x_{0}-x\right)^{2}+\left(y_{0}-y\right)^{2}}{2 \sigma_{b 2}{ }^{2}}}
\end{aligned}
$$

where $w_{b 1}$ and $w_{b 2}$ represent the amplitudes of two Gaussian weight components, whose standard deviations are $\sigma_{b 1}$ and $\sigma_{b 2}$ respectively, in $W_{b}\left(x_{x}, y_{0}, x_{0, y_{0}}\right)$.

Signal converge into bipolar cell $(x, y)$ is:

$$
\begin{aligned}
\boldsymbol{I}_{b}(x, y)= & \sum_{x_{0}, y_{0} \in R_{b, c}} W_{b}\left(x, y ; x_{0}, y_{0}\right) \boldsymbol{o}_{p}\left(x_{0}, y_{0}\right) \\
& -\sum_{x_{0}, y_{0} \in R_{b, s}} W_{b}\left(x, y ; x_{0}, y_{0}\right) \boldsymbol{o}_{h}\left(x_{0}, y_{0}\right)
\end{aligned}
$$

where $R_{b_{b} c}$ and $R_{b_{j} g}$ represent the center and surround region of center-surround antagonistic receptive field, $\boldsymbol{o}_{\mathrm{p}}\left(x_{0}, y_{0}\right)$ and $\boldsymbol{O}_{\hat{h}}\left(x_{0 x} y_{0}\right)$ represent respectively the output signals from PCs and HCs located at $\left(x_{0}, y_{0}\right)$ in CSARF.

The response dynamics of retina $\mathrm{BCs}$ to stimulus is described as following equation[29]:

$$
\theta_{b} \frac{d}{d t} o_{b}\left(x_{v} y_{s} t\right)+O_{b}\left(x_{v} y_{s} t\right)=I_{b}\left(x_{v} y_{v} t\right)
$$


where $\boldsymbol{O}_{b}\left(x_{x}, y, t\right)$ and $I_{b}\left(x_{x}, y, t\right)$ represent the membrane potential of a bipolar cell $\left(x_{x} y\right)$ and its input signal respectively, and $\theta_{b}$ is a time constant.

\section{Spatio-Temporal Properties of Starburst Amacrine Cells}

Amacrine cells are served to integrate, modulate and interpose a temporal domain to the visual message presented to the ganglion cell. Experiments [5][15] show that SAC are wired to suppress the visual response of DSGCs through presynaptic inhibition of a bipolar terminal.

The weight distribution in receptive field of SACs is described as:

$$
W_{S A C}\left(x, y ; x_{0}, y_{0}\right)=A_{1} e^{-\frac{\left(x_{0}-x\right)^{2}+\left(y_{0}-y\right)^{2}}{2 \sigma_{a 1}^{2}}}-A_{2} e^{-\frac{\left(x_{0}-x\right)^{2}+\left(y_{0}-y\right)^{2}}{2 \sigma_{a 2}^{2}}}
$$

Where $A_{1}$ and $A_{2}$ are respectively the amplitude of two components, and $\sigma_{a 1}$ and $\sigma_{a 2}$ are standard deviation of Gaussian shape components.

Inputs convergence into a SAC can be represented in following equation:

$$
I_{S A C}(x, y)=\sum_{\left(x_{0}, y_{0}\right) \in c e n t r e} W_{S A C}\left(x, y ; x_{0}, y_{0}\right) O_{b}-\sum_{\left(x_{0}, y_{0}\right) \in \text { surr }} W_{S A C}\left(x, y ; x_{0}, y_{0}\right) O_{S A C}
$$

Where centre and suur respectively represent the centre and surround region in receptive field of SACs.

The dynamic property of SACs is represented as [16]:

$$
\frac{d}{d t} O_{S A C}(t)=-A O_{S A C}(t)+\left[B-O_{S A C}(t)\right] e(t)-\left[D+O_{S A C}(t)\right] j(t)+\sum_{k=1}^{n} W_{S A C} O_{k}(t)
$$

Where $e(t)$ and $i(t)$ respectively represent the excitatory and inhibitory inputs. $O_{S A C}$ represents the membrane potential of SAC locates at $(x, y)$, while $O_{k}$ is the membrane potential of the neuronal element which makes lateral connections with SAC element $(x, y), A, B$ and $D$ are constants for respectively represent the rate of potential decay, the saturation levels for the excitatory and inhabitory inputs, $W_{S A C}$ is the lateral connections to the neuron of interest, i.e. $\operatorname{SAC}(x, y)$.

\section{E. Spatio-Temporal Properties of Directionally Selective Ganglion Cells}

DSGCs signal the direction of image motion across their receptive fields by firing action potentials in a 'preferred' direction, other than in the opposite 'null' direction. A key circuit module of retinal DSGCs is a spatially asymmetric inhibitory input from starburst amacrine cells [17-19].

The preferred direction of the cells and the strength of the directional tuning of the DSGCs can be calculated from responses to stimuli in each of stimulus directions evenly spanning $360^{\circ}$. With directional tuning data, the response $R$ of a DSGC to stimulus from the direction $\chi$ can be described by a von Mises distribution [20] as:

$$
R(\chi)=R_{\max } e^{\left(\kappa \cos \left(\frac{(\chi-\mu) \pi}{180}\right)\right)} / e^{\kappa}
$$

Where $R_{\max }$ is the maximum response, $\mu$ becomes the preferred direction in degrees, and $\kappa$ is the concentration parameter, which accounts for the width of the directional tuning.

The weight distribution of excitory component and inhibitory component in DSGCs receptive field can be represented respectively as:

$$
\begin{aligned}
& W_{\text {gang }}^{\text {exc }}\left(x, y ; x_{0}, y_{0}\right)=\frac{1}{\sqrt{2 \pi \sigma_{g}}} e^{-\frac{\left(x-x_{0}\right)^{2}+\left(y-y_{0}\right)^{2}}{\sigma_{g}^{2}}}, x_{0}, y_{0} \in R_{\text {exe }} \\
& W_{\text {gang }}^{\text {inh }}\left(x, y ; x_{0}, y_{0}\right)=\frac{1}{\sqrt{2 \pi \sigma_{g 1} \sigma_{g 2}}} e^{-\frac{\left(x_{0}-x\right)^{2}}{\sigma_{g 1}^{2}}+\frac{\left(y_{0}-y\right)^{2}}{\sigma_{g 2}^{2}}}, x_{0}, y_{0} \in R_{\text {inh }}
\end{aligned}
$$

Where $\sigma_{g}, \sigma_{g 1}$ and $\sigma_{g 2}$ represent the standard deviation of two DSGCs receptive field weight distributions respectively. $R_{\text {exe }}$ and $R_{\text {inh }}$ represent a symmetric and an asymmetric region in receptive field of $\operatorname{DSGC}(x, y)$ respectively.

Inputs convergence to a $\operatorname{DSGC}(x, y)$ is:

$$
I_{\text {gang }}(x, y)=\sum_{x_{0}, y_{0} \in R_{\text {ac }}} W_{\text {gang }}^{\text {exc }}\left(x, y ; x_{0}, y_{0}\right) O_{b}+\sum_{x_{0}, y_{0} \in R_{\text {inh }}} W_{\text {gang }}^{\text {inh }}\left(x, y ; x_{0}, y_{0}\right) O_{S A C}
$$

Where $O_{b}$ and $O_{S A C}$ represent the output from bipolar cells in $R_{\text {exe }}$ and amacrine cells in $R_{i n h}$.

TABLE I Parameters of the Proposed Retina Neural Network

\begin{tabular}{|c|c|c|c|}
\hline Cell Type & $\begin{array}{c}\text { Width of } \\
\text { RF (pixels) }\end{array}$ & $\begin{array}{c}\text { Standard } \\
\text { deviation of } \\
\text { Gaussians }\end{array}$ & Other parameters \\
\hline Photoreceptor cells & Null & Null & $\mu_{p}=0.5$ \\
\hline Horizontal cell & 27 & $\sigma_{h}=4.5$ & $\lambda=0.03, w_{h}=1$ \\
\hline $\begin{array}{c}\text { Bipolar cell } \\
\text { Starburst amacrine } \\
\text { cells }\end{array}$ & 5 & $\sigma_{b 1}=0.6, \sigma_{b 2}=5.4$ & $w_{b 1}=w_{b 2}=1$ \\
\hline $\begin{array}{c}\text { Ganglion cell } \\
\text { (15 }\end{array}$ & 21 & $\begin{array}{c}\sigma_{a 1}=0.6, \sigma_{a 2}=5.4 \\
\sigma_{g 1}=0.498 ;\end{array}$ & $\begin{array}{c}A=1 ; B=2 ; D=0.5 ; \\
A_{1}=1 ; A_{2}=11.2\end{array}$ \\
\hline
\end{tabular}


TABLE II Mean Square Error (MSE) of Motion Velocity Matrices Detected by Using Different Methods

\begin{tabular}{|c|c|c|c|c|c|c|c|c|c|c|c|c|c|c|}
\hline \multirow{2}{*}{\multicolumn{2}{|c|}{$\begin{array}{c}\text { Motion } \\
\text { direction }\end{array}$}} & \multicolumn{13}{|c|}{ Frames } \\
\hline & & 1 & 2 & 3 & 4 & 5 & 6 & 7 & 8 & 9 & 10 & 11 & 12 & 13 \\
\hline \multirow{2}{*}{$0^{\circ}$} & $\begin{array}{l}\text { Retina } \\
\text { model }\end{array}$ & 0.0525 & 0.0525 & 0.0525 & 0.0520 & 0.0501 & 0.0501 & 0.0501 & 0.0501 & 0.0501 & 0.0501 & 0.0501 & 0.0501 & $\begin{array}{l}0.050 \\
1 \\
\end{array}$ \\
\hline & $\begin{array}{l}\text { Optical } \\
\text { flow }\end{array}$ & 0.0532 & 0.0532 & 0.0532 & 0.0532 & 0.0532 & 0.0532 & 0.0532 & 0.0532 & 0.0532 & 0.0532 & 0.0532 & 0.0532 & $\begin{array}{l}0.072 \\
2 \\
\end{array}$ \\
\hline \multirow{2}{*}{$45^{\circ}$} & $\begin{array}{l}\text { Retina } \\
\text { model }\end{array}$ & 0.0742 & 0.0742 & 0.0742 & 0.0730 & 0.0723 & 0.0723 & 0.0723 & 0.0724 & 0.0724 & 0.0724 & 0.0724 & 0.0724 & $\begin{array}{l}0.072 \\
4\end{array}$ \\
\hline & $\begin{array}{l}\text { Optical } \\
\text { flow }\end{array}$ & 0.0750 & 0.0750 & 0.0750 & 0.0750 & 0.0750 & 0.0750 & 0.0750 & 0.0750 & 0.0750 & 0.0750 & 0.0750 & 0.0750 & $\begin{array}{l}0.102 \\
4\end{array}$ \\
\hline \multirow{2}{*}{$90^{\circ}$} & $\begin{array}{l}\text { Retina } \\
\text { model }\end{array}$ & 0.0525 & 0.0525 & 0.0525 & 0.0520 & 0.0519 & 0.0519 & 0.0519 & 0.0519 & 0.0519 & 0.0519 & 0.0519 & 0.0519 & $\begin{array}{l}0.051 \\
9\end{array}$ \\
\hline & $\begin{array}{l}\text { Optical } \\
\text { flow }\end{array}$ & 0.0577 & 0.0577 & 0.0577 & 0.0577 & 0.0577 & 0.0577 & 0.0577 & 0.0577 & 0.0577 & 0.0577 & 0.0577 & 0.0577 & $\begin{array}{l}0.073 \\
2\end{array}$ \\
\hline \multirow{2}{*}{$135^{\circ}$} & $\begin{array}{l}\text { Retina } \\
\text { model }\end{array}$ & 0.0742 & 0.0742 & 0.0742 & 0.0731 & 0.0731 & 0.0731 & 0.0731 & 0.0731 & 0.0731 & 0.0731 & 0.0731 & 0.0731 & $\begin{array}{l}0.073 \\
1\end{array}$ \\
\hline & $\begin{array}{l}\text { Optical } \\
\text { flow }\end{array}$ & 0.0750 & 0.0750 & 0.0750 & 0.0750 & 0.0750 & 0.0750 & 0.0750 & 0.0750 & 0.0750 & 0.0750 & 0.0750 & 0.0750 & $\begin{array}{l}0.102 \\
4\end{array}$ \\
\hline \multirow{2}{*}{$180^{\circ}$} & $\begin{array}{l}\text { Retina } \\
\text { model }\end{array}$ & 0.0525 & 0.0525 & 0.0525 & 0.0520 & 0.0501 & 0.0501 & 0.0501 & 0.0501 & 0.0501 & 0.0501 & 0.0501 & 0.0501 & $\begin{array}{l}0.050 \\
1 \\
\end{array}$ \\
\hline & $\begin{array}{l}\text { Optical } \\
\text { flow }\end{array}$ & 0.0532 & 0.0532 & 0.0532 & 0.0532 & 0.0532 & 0.0532 & 0.0532 & 0.0532 & 0.0532 & 0.0532 & 0.0532 & 0.0532 & $\begin{array}{l}0.072 \\
2\end{array}$ \\
\hline
\end{tabular}

\section{Experimental Results and Corresponding Analysis}

To evaluate the effect of the proposed retina model on motion detection, quantitative analysis and comparasion with other method (here it is optical flow based algorithm) are both accomplished. For achieving this, the criterion on performance evaluation of motion detection algorithms will be firstly given in this section. Obviously, for a method of object motion detection, accuracy, universality and timeliness are the three most important factors that should be used to construct a formula for measuring system performance on motion detection. The following expression is one of this kind of representations:

$$
\hat{e}_{v} \sqrt{\frac{1}{H \cdot V} \sum_{i=1}^{H} \sum_{j=1}^{V}\left(E_{h}^{2}(i, j)+E_{v}^{2}(i, j)\right)}
$$

Where $\hat{e}_{v}$ denotes the mean square error (MSE) of detected motion velocity on real one, $H$ and $V$ are respectively the pixels on horizontal and vertical direction, accordingly $E_{h}$ and $E_{v}$ are respectively error matrices for representing the horizontal and vertical component of velocity vector field, and they read:

$$
\begin{gathered}
E_{v}=R_{v}-\frac{\hat{R}_{v}}{\max _{1 \leq i \leq H, 1 \leq j \leq V}\left(\sqrt{\hat{R}_{h}{ }^{2}(i, j)+\hat{R}_{v}{ }^{2}(i, j)}\right)} \\
E_{v}=R_{v}-\frac{\hat{R}_{v}}{\max _{1 \leq i \leq H, 1 \leq j \leq V}\left(\sqrt{\hat{R}_{h}{ }^{2}(i, j)+\hat{R}_{v}{ }^{2}(i, j)}\right)}
\end{gathered}
$$

Where $\hat{R}_{h}$ and $\hat{R}_{v}$ are respectively the matrices for representing the horizontal and vertical component of velocity vector field that that have been detected by using certain motion detection algorithm, while $R_{h}$ and $R_{v}$ are matrices for real motion velocity components. Table II shows the mean square error (MSE) of motion velocity matrices detected by using different methods (the proposed retina model vs. Optical Flow).

As can be seen from the above table, the two methods show similar motion sensitivities as well as good accuracy on the direction of $0^{\circ}, 90^{\circ}, 180^{\circ}$ and $315^{\circ}$. And generally, the proposed retina model shows better performance on motion detection than the traditional optical flow based algorithms. Or it has a lower value of MSE than that with traditional optical flow based algorithms.

\section{Conclusions}

In this paper, a bioinspired neural model for detecting object motion based on retina computational machanism is proposed based on synthesizing those representative works on modeling retina and incorporating some latest findings on retina mechanisms. To understand more in-depth the retina machanism of motion detection, the spatio-temporal properties of each type of neurons in the retina motion pathway is also mathematically analyzed. For evaluating the model performance on motion detection, a set of experiments and quantitative analysis of the experimental results are accomplished. The experimental results show that this proposed model can be used to detect object motion effectively.

\section{Acknowledgment}

The authors would like to thank the Hunan Natural Science Foundation, China, and the China Hunan Provincial Science \& Technology Department for their financial supports.

\section{References}

[1] Wei Wei, Aaron M. Hamby, Kaili Zhou \& Marla B. Feller, "Development of asymmetric inhibition underlying direction selectivity 
in the retina", Nature, 469(1): 402-406, 2011.

[2] K. Boahen, "A retinomorphic chip with parallel pathways: Encoding ON, OFF, INCREASING, and DECREASING visual signals", Analog Integr. Circuits Signal Process., 30(2):121-135, 2002.

[3] K. A. Zaghloul, K. A. Boahen, "Optic nerve signals in a neuromorphic chip I: Outer and inner retina models", IEEE Trans. Biomed. Eng., 51(4):657-666, 2004.

[4] Barranco F, Díaz J, Ros E, del Pino B., "Visual system based on artificial retina for motion detection", IEEE Tran. Sys. Man Cybern B Cybern., 39(3):752-62, 2009.

[5] Baccus, Stephen A., Bence P. Ölveczky, Mihai Manu, Markus Meister, "A retinal circuit that computes object motion", The Journal of Neuroscience, 28(27): 6807-6817, 2008.

[6] Keisuke Yonehara, Kamill Balint, Masaharu Noda, Georg Nagel, Ernst Bamberg \& Botond Roska, "Spatially asymmetric reorganization of inhibition establishes a motion-sensitive circuit", Nature, 469(1): 407410, 2011.

[7] Taylor W. R., He S., Levick W. R., Vaney D. I., "Dendritic computation of direction selectivity by retinal ganglion cells", Science, 289: 23472350,2000

[8] Grzywacz N. M., Amthor F. R., Mistler L. A., "Applicability of quadratic and threshold models to motion discrimination in the rabbit retina", Biol. Cybern., 64: 41-49, 1990.

[9] Koch C., Poggio T., Torre V., "Retinal ganglion cells: A functional interpretation of dendritic morphology", Philos. Trans. R. Soc. Lond. B Biol. Sci., 298:227-264,1982.

[10] Germán A. Enciso, Michael Rempe, Andrey V. Dmitriev, Konstantin E. Gavrikov, David Terman, Stuart C. Mangel, "A model of direction selectivity in the starburst amacrine cell network", J. Comput. Neurosci., 28:567-578, 2010.

[11] Shelley I. Fried, Thomas A. Munch, Frank S. Werblin, "Mechanisms and circuitry underlying directional selectivity in the retina", NATURE, 420(28):411-413, 2002.

[12] Burkhardt D. A., Fahey P. K., "Contrast enhancement and distributed encoding by bipolar cells in the retina", J. Neurophysiol ., 80:1070 $1081,1998$.

[13] Demb J. B., Zaghloul K., Haarsma L., Sterling P., "Bipolar cells contribute to nonlinear spatial summation in the brisk-transient $(\mathrm{Y})$ ganglion cell in mammalian retina", J. Neurosci., 21:7447-7454, 2001.

[14] Olveczky B. P., Baccus S. A., Meister M., "Retinal adaptation to object motion", Neuron, 56:689 -700, 2007.

[15] Murat Saglam, Yuki Hayashida, Nobuki Murayama, "A retinal circuit model accounting for wide-field amacrine cells", Cogn. Neurodyn., 3:15-32, 2009.

[16] Fried S. I., Munch T. A., Werblin F. S., "Mechanisms and circuitry underlying directional selectivity in the retina", Nature, 420: 411-414, 2002.

[17] Euler T., Detwiler P. B., Denk W., "Directionally selective calcium signals in dendrites of starburst amacrine cells", Nature, 418:845-852, 2002.

[18] Lee S., Zhou Z. J., "The synaptic mechanism of direction selectivity in distal processes of starburst amacrine cells", Neuron, 51: 787-799, 2006.

[19] Nicholas Oesch, Thomas Euler, W. Rowland Taylor, "DirectionSelective Dendritic Action Potentials in Rabbit Retina", Neuron, 47(5):739-750, 2005. 